\title{
Influence of environmental factors on the detection of blood in sheep faeces using visible-near-infrared spectroscopy as a measure of Haemonchus contortus infection
}

\author{
Elise A. Kho ${ }^{1}$, Jill N. Fernandes ${ }^{1}$, Andrew C. Kotze ${ }^{2}$, Glen P. Fox ${ }^{3}$, Maggy T. Sikulu-Lord ${ }^{4}$, Anne M. Beasley ${ }^{5}$,
} Stephen S. Moore ${ }^{1}$ and Peter J. James ${ }^{1 *}$

\begin{abstract}
Background: Existing diagnostic methods for the parasitic gastrointestinal nematode, Haemonchus contortus, are time consuming and require specialised expertise, limiting their utility in the field. A practical, on-farm diagnostic tool could facilitate timely treatment decisions, thereby preventing losses in production and flock welfare. We previously demonstrated the ability of visible-near-infrared (Vis-NIR) spectroscopy to detect and quantify blood in sheep faeces with high accuracy. Here we report our investigation of whether variation in sheep type and environment affect the prediction accuracy of Vis-NIR spectroscopy in quantifying blood in faeces.

Methods: Visible-NIR spectra were obtained from worm-free sheep faeces collected from different environments and sheep types in South Australia (SA) and New South Wales, Australia and spiked with various sheep blood concentrations. Spectra were analysed using principal component analysis (PCA), and calibration models were built around the haemoglobin $(\mathrm{Hb})$ wavelength region (387-609 nm) using partial least squares regression. Models were used to predict $\mathrm{Hb}$ concentrations in spiked faeces from SA and naturally infected sheep faeces from Queensland (QLD). Samples from QLD were quantified using Hemastix ${ }^{\circledR}$ test strip and FAMACHA@ diagnostic test scores.

Results: Principal component analysis showed that location, class of sheep and pooled versus individual samples were factors affecting the $\mathrm{Hb}$ predictions. The models successfully differentiated 'healthy'SA samples from those requiring anthelmintic treatment with moderate to good prediction accuracy (sensitivity 57-94\%, specificity 44-79\%). The models were not predictive for blood in the naturally infected QLD samples, which may be due in part to variability of faecal background and blood chemistry between samples, or the difference in validation methods used for blood quantification. PCA of the QLD samples, however, identified a difference between samples containing high and low quantities of blood.

Conclusion: This study demonstrates the potential of Vis-NIR spectroscopy for estimating blood concentration in faeces from various types of sheep and environmental backgrounds. However, the calibration models developed here did not capture sufficient environmental variation to accurately predict $\mathrm{Hb}$ in faeces collected from environments different to those used in the calibration model. Consequently, it will be necessary to establish models that incorporate samples that are more representative of areas where $H$. contortus is endemic.
\end{abstract}

*Correspondence: p.james1@uq.edu.au

${ }^{1}$ The Queensland Alliance for Agriculture and Food Innovation, The

University of Queensland, St. Lucia, QLD 4067, Australia

Full list of author information is available at the end of the article

c) The Author(s) 2020. This article is licensed under a Creative Commons Attribution 4.0 International License, which permits use, sharing, adaptation, distribution and reproduction in any medium or format, as long as you give appropriate credit to the original author(s) and the source, provide a link to the Creative Commons licence, and indicate if changes were made. The images or other third party material in this article are included in the article's Creative Commons licence, unless indicated otherwise in a credit line to the material. If material is not included in the article's Creative Commons licence and your intended use is not permitted by statutory regulation or exceeds the permitted use, you will need to obtain permission directly from the copyright holder. To view a copy of this licence, visit http://creativeco mmons.org/licenses/by/4.0/. The Creative Commons Public Domain Dedication waiver (http://creativecommons.org/publicdomain/ zero/1.0/) applies to the data made available in this article, unless otherwise stated in a credit line to the data. 
Keywords: Haemonchus contortus, Visible-near infrared spectroscopy, Haemoglobin, Blood, Faecal analysis, Gastrointestinal nematodes

\section{Background}

Gastrointestinal nematodes (GIN) cause significant disease and economic losses in grazing animals [1]. Haemonchus contortus is one of the most damaging GIN species, affecting the health and welfare of small ruminant livestock worldwide [2, 3]. Warm, moist conditions favour the survival of $H$. contortus larvae; however, larvae can also survive in relatively cold and dry conditions by suspending development in their host during winter (termed hypobiosis) and continuing their development and transmission in the following spring [4]. In Australia, $H$. contortus is most prevalent in south-eastern Queensland and northern New South Wales [5], although the distribution of $H$. contortus has been expanding to more southern parts of the country [6]. In favourable conditions, female $H$. contortus are capable of laying up to 10,000 eggs daily, resulting in rapid and extensive pasture contamination, thus increasing flock exposure to the parasite $[7,8]$. Animals infected with $H$. contortus can suffer blood loss of up to $30 \mathrm{~mL}$ daily in severe cases $[9,10]$. Blood loss in sheep typically occurs from blood ingested by the nematode and blood leakage into the sheep gut due to damage sustained through nematode attachment [10].

Slaughter of sheep to quantify nematodes is the gold standard method for the precise estimation of parasite burden in sheep. This method is also often required to demonstrate anthelmintic efficacy for the registration of new control products, but the need to euthanase sheep makes it an impractical method for use on farms [11]. Faecal worm egg count (FWEC) is the method currently recommended for GIN monitoring by the World Association for the Advancement of Veterinary Parasitology and the one used by many sheep producers $[12,13]$. The most commonly used FWEC technique, the McMaster method, is known to have a relatively low analytical sensitivity of 10-50 eggs per gram of faeces (epg) and high variability between subsamples [14-17]. It has been reported that the presence of blood in faeces from infected sheep can be detected as early as 11 days after the initial infection, whereas $H$. contortus eggs begin to occur in faeces at approximately 18 days post-infection [2, 9]. Therefore, for new infections, the detection of blood or haemoglobin $(\mathrm{Hb})$ in the faeces may enable earlier diagnosis of $H$. contortus infections in sheep than with FWEC. Another potential advantage of blood (haemoglobin) over eggs for diagnosis is that blood is more evenly distributed within the faeces, resulting in less variability between samples, although this needs to be confirmed.

Several tools based on blood loss have been developed for the diagnosis of $H$. contortus infections. One of these, the Haemonchus Dipstick Test, which is no longer commercially available, quantified the amount of faecal occult blood (FOB; blood that is not visibly apparent) present in sheep faeces $[18,19]$. The FOB reagent strips Hemastix $^{\circledR}$ (Bayer HealthCare LLC, Bayer AG, Leverkusen, Germany) were used to detect the presence of $\mathrm{Hb}$ peroxidase activity in faeces as an index of the level of worm infection present. Given that peroxidase activity can also be found in plant materials, a strict boiling time of 20 min was necessary to denature the peroxidase and ensure accuracy of the test $[18,20]$. The dipstick test used a score range of $1-5$, with the manufacturer's manual stating that a score of 1 indicates a negative result and a score of 5 indicates a concentration of $\geq 200$ blood cells/ $\mu \mathrm{L}$, suggesting the presence of a heavy $H$. contortus infection requiring immediate anthelmintic treatment. Detailed interpretation of the test result was challenging, however, as each score corresponded to a wide range of $\mathrm{Hb}$ concentrations [21]. Additionally, FOB test kits such as Hemastix ${ }^{\circledR}$ have been found to be less sensitive when used with samples from mixed-parasite infections [21].

The FAMACHA $\odot$ method is a five-point scoring system that can be used in sheep yards by a trained observer to assess the level of anaemia of a given animal [22]. Relatively good sensitivity of the FAMACHA® method has been reported in various regions for detecting anaemic animals ( $>50 \%$ for sheep and $>89 \%$ for goats) [23-27]. However, despite the relative ease of use and low cost of FAMACHA@, a drawback of this system is that it is relatively labour intensive and relies heavily on the operator's experience, which can lead to inconsistent results both within and across sheep management systems [23]. In addition, production losses can still occur before scores indicative of the need for anthelmintic treatment are obtained, resulting in economic losses for sheep producers $[27,29,30]$. An automated method which uses scanning of the third eyelid (palpebral conjunctiva) of the animal and image analysis to predict $\mathrm{Hb}$ levels has recently been validated in calves and could potentially improve the accuracy of prediction by reducing the operator variability associated with the FAMACHA๑ method [28]. However, whether this method is applicable to the diagnosis of helminth infections in sheep or could 
provide earlier prediction of the need to treat is yet to be determined.

Previous studies evaluating the accuracy of FOB test kits were performed based on the measurement of sheep blood collected by jugular venipuncture, rather than by direct measurement of FOB in faeces [11, 21]. In these studies, results from packed cell volume and $\mathrm{Hb}$ concentrations obtained from whole blood samples for sheep infected with $H$. contortus were compared with the predictions from the FOB test kits. It can be challenging to evaluate the accuracy of $\mathrm{Hb}$ assessed using FOB test kits, as there may not be a direct relationship between the changes in haematocrit levels in the host blood and the presence of blood in the faeces [21]. Furthermore, there is no quantitative method available for the direct measurement of $\mathrm{Hb}$ concentrations in sheep faeces.

Faecal analyses using near-infrared (NIR) spectroscopy have been previously used to evaluate diet quality in ruminants and to monitor their health and welfare [31-35]. Faecal NIR spectroscopy has also been applied to estimate tick burdens in cattle and horses through the prediction of stress [36] and internal parasite burdens through the prediction of FWEC [35]. The use of NIR spectroscopy for chemical analysis in animals has continued to increase due to its wide range of applications, low processing time, low cost, non-invasiveness, bulk-sampling capacity and the ability to measure samples under different conditions [37-39]. Importantly, it is possible to use a single NIR spectral scan of a sample to determine several chemical attributes simultaneously. However, spectroscopists must structure and design calibration models carefully to focus on specific chemical attributes related to their particular topic of interest.

The ultimate aim of a test to determine the need for anthelmintic treatment is to estimate the total worm count of $H$. contortus present in the abomasum of sheep. This is most commonly done using FWEC but, as noted, FWEC may underestimate the worm burden, given that nematodes may be present for up to 7 days before they produce eggs [40, 41]. Furthermore, methods involving the assessment of anaemia may be affected by the experience of the operator [23, 24]. Given that there are currently no tests available to directly measure sheep blood content in faeces, it is particularly challenging to determine reference values for the validation of a prediction model-in our case, the validation of a calibration model based on blood content of the faeces. For these reasons, the calibration models reported here were based on faecal samples spiked with known concentrations of sheep blood.

We previously reported the potential of visible (Vis)NIR spectroscopy for detecting blood in sheep faeces, as an indicator of $H$. contortus infection [42]. In that study, we developed calibration models within the wavelength region of 400-600 nm to measure blood in faeces collected from a single sheep and found that the prediction precision was lowest with the lower $\mathrm{Hb}$ concentrations, likely due to the lack of physical, chemical and environmental variation in the faecal samples in the calibration model. In the study reported here, we investigated the predictability of calibration models built using faeces collected from various locations free from $H$. contortus and spiked with known amounts of blood, for the prediction of $\mathrm{Hb}$ concentrations in both blood-spiked and naturally infected sheep faeces.

\section{Methods}

\section{Faecal sample collection and faeces-blood preparation}

In order to obtain blood-free field-collected sheep faeces from a range of different environments and sheep backgrounds, faecal samples were collected during the winter-spring of 2019 (August-October) from multiple sheep properties in South Australia (SA) known to be free from $H$. contortus infection (based on previous worm egg counts and larval differentiations). Faecal samples were collected both as individual samples from single sheep and as pooled samples where ten individual samples of faeces were combined and later subsampled for Vis-NIR analysis. Faecal consistency was described as very dry pellets (VDP), dry pellets (DP), dry aggregates (DA) moist pellets (MP) and very moist pellets (VMP). Fresh samples were collected off the ground from ewes, rams, lambs $(<1$ year old $)$ and hoggets $(1-2$ years old $)$ into separate sample bags shortly after defecation. Faecal samples were transported on ice to Brisbane, QLD and stored at $-20{ }^{\circ} \mathrm{C}$ until further analysis. Faecal samples were also collected from Armidale, New South Wales (NSW) as described in Kho et al. [42]. These samples were collected from a single uninfected sheep kept in an animal house, fed a diet consisting of ground wheat grain and ground lucerne hay. For the purpose of this study, samples from this animal are defined as the uninfected control samples. All animal procedures were approved by the FD McMaster Animal Ethics Committee, CSIRO Agriculture and Food (Animal Ethics Approval Number AEC 18/09).

The frozen samples were thawed over a 2-h period, and the weights of faeces from each sample were recorded. Faeces from each collection bag was thoroughly homogenised, and 10-g subsamples were mixed with various concentrations of diluted defibrinated sheep blood. Faeces-blood samples and controls were sandwiched between two sheets of polyethylene film (Woolworths Australia, Bella Vista, NSW, Australia) and thoroughly dispersed by rolling and folding each sample multiple times to form a 'slab' $(10 \times 20 \mathrm{~cm})$ before measurement at 
randomly selected points with a Vis-NIR spectrometer [43].

Defibrinated sheep blood used throughout the study was purchased from a commercial supplier (Serum Australis, Manilla, NSW, Australia) and serially diluted with distilled water at the ratio of 1:2. Each slab consisted of $10 \mathrm{~g}$ of faeces mixed with $5 \mathrm{~mL}$ of diluted blood to provide final concentrations of $0,2,2.3,4,4.25,4.5,8,8.5$ and $9 \mu \mathrm{g} \mathrm{Hb} / \mathrm{mg}$ faeces. Control samples were prepared by adding $5 \mathrm{~mL}$ water to $10 \mathrm{~g}$ faeces.

Faeces were also collected from The University of Queensland (UQ) Gatton campus, QLD, where H. contortus is endemic, on 2 days in February and March 2020, respectively. A total of $221 \mathrm{~mm}$ of rainfall had been recorded over the preceding 2 months, providing favourable conditions for $H$. contortus transmission [44, 45]. These samples were included to represent samples collected from a different region known to be prone to $H$. contortus infections. Sheep faeces were collected off the ground shortly after defecation and were placed into individual sample bags. Sheep identification (ID) was recorded on all collection bags. The FAMACHA $\odot$ scores and history of anthelmintic treatments for the corresponding sheep ID were obtained and recorded on the same day as faeces collection by trained and experienced veterinarians at UQ Gatton, QLD. Faecal samples from each bag were homogenised and subsamples processed within $24 \mathrm{~h}$ of collection using the McMaster method to determine FWEC and Hemastix ${ }^{\circledR}$ to estimate the amount of blood present in the faeces. The remainder of each sample was stored at $-20{ }^{\circ} \mathrm{C}$ until further analysis with Vis-NIR spectroscopy. Prior to spectroscopic measurement, the faecal samples were thawed for $2 \mathrm{~h}$ and later scanned as a slab $(10 \times 20 \mathrm{~cm})$ sandwiched between two sheets of polyethylene film. All animal procedures here were approved by the UQ Animal ethics committee (Animal Ethics Approval Number AEC: SVS/452/17).

\section{Calibration and validation dataset}

As the diagnosis of $H$. contortus infection using FWEC and Hemastix ${ }^{\circledR}$ is typically performed by pooling faeces collected from multiple individual animals $[18,46]$, we determined the effects of using pooled or individual samples for building calibration models. Two calibration models were built using faeces collected from different locations in SA and NSW. Model 1 consisted of faeces from individual sheep, while Model 2 included faeces from both individual and pooled samples. Table 1 shows the description of samples included in each model.
Four groups of samples were prepared and measured to form the validation datasets. The $\mathrm{Hb}$ concentrations of the faecal samples used in the validation datasets were $0,2,2.3,4,4.25,4.5,8,8.5$ and $9 \mu \mathrm{g} \mathrm{Hb} / \mathrm{mg}$ faeces. For samples collected as 'individual' sample type, each bag of faeces was subsampled, spiked with blood and scanned as individual samples for each $\mathrm{Hb}$ concentrations. For samples designated as 'pooled', replicates of each faeces were subsampled and spiked with blood before scanning using Vis-NIR spectrometer. To include variations that may be present in future validations, a randomly selected portion of faecal samples included in Models 1 and 2 were mixed with various concentrations of $\mathrm{Hb}$ and scanned as a slab on separate days using the Vis-NIR spectrometer (Val1 and Val2). Faecal samples were collected from the Lower North and Yorke Peninsula in SA to represent locations not included in the models as validation dataset 3 (Val3). Faeces were also collected from UQ Gatton, QLD as validation dataset 4 (Val4) to represent samples from different regions with naturally occurring $H$. contortus infections. Table 2 shows detailed descriptions of the validation datasets used in this study.

\section{Spectral acquisition with Vis-NIR spectroscopy}

A Felix F-750 portable Vis-NIR spectrometer (Felix Instruments, Camas, WA, USA), equipped with a xenon tungsten lamp as the light source, was used to collect spectra throughout this study. The Vis-NIR spectra of blood in sheep faeces were obtained within the wavelength range of $300-1200 \mathrm{~nm}$ at a spectral resolution of between 8 and $13 \mathrm{~nm}$ and data resolution of $3 \mathrm{~nm}$. Each spectrum was obtained using 32 scans. Faeces-blood mixture slabs were placed on top of the scanning window of the Felix F-750 spectrometer, and a white Teflon disc was placed above the slab to augment the signal-to-noise ratio [42]. Spectra were acquired randomly between prepared faeces slabs and across ten random scanning points on each faeces-blood mixture slab using a randomised list generated from Microsoft Excel (2016 version; Microsoft Corp., Redmond, WA, USA). The average of ten spectra from each slab was calculated and used for the development of the VisNIR calibration models.

\section{Faecal worm egg count, Hemastix ${ }^{\circledR}$ and FAMACHA®}

Faecal worm egg counts were performed using the modified McMaster method [12]. Briefly, faeces from each collection bag were homogenised, and a subsample of $2 \mathrm{~g}$ faeces was mixed with $16 \mathrm{~mL}$ of concentrated salt solution $\left(\mathrm{MgSO}_{4}\right.$, specific gravity $\left.[\mathrm{SG}]=1.18\right)$. The faecal slurry was strained through a tea strainer to remove large 
Table 1 Description of samples included in the calibration models built for prediction of haemoglobin in sheep faeces using a visible-near-infrared spectrometer

\begin{tabular}{|c|c|c|c|c|}
\hline Model & Sample origin & Sample type & Class of sheep & $n$ \\
\hline \multirow[t]{6}{*}{1} & Adelaide Hills, SA & Individual & Ewes & 44 \\
\hline & Armidale, NSW & Single animal & Wether (uninfected control) & 6 \\
\hline & Fleurieu Peninsula, SA & Individual & Ewes and lambs & 24 \\
\hline & Currency Creek, SA & Individual & Ewes & 10 \\
\hline & Eyre Peninsula, SA & Individual & Ewes and hoggets & 35 \\
\hline & Total & & & 119 \\
\hline \multirow[t]{8}{*}{2} & Adelaide Hills, SA & Individual & Ewes & 44 \\
\hline & Armidale, NSW & Single animal & Wether (uninfected control) & 6 \\
\hline & Mid-North 1, SA & Pooled & Ewes & 22 \\
\hline & Mid-North 2, SA & Pooled & Ewes & 13 \\
\hline & Fleurieu Peninsula, SA & Individual & Ewes and lambs & 24 \\
\hline & Currency Creek, SA & Individual & Ewes & 10 \\
\hline & Eyre Peninsula, SA & Individual & Ewes and hoggets & 35 \\
\hline & Total & & & 153 \\
\hline
\end{tabular}

NSW New South Wales, SA South Australia

Table 2 Description of samples included in the validation datasets

\begin{tabular}{|c|c|c|c|c|}
\hline Validation dataset & Sample origin & Sample type & Class of sheep & $n$ \\
\hline \multirow[t]{5}{*}{ Val1 } & Mid-North 1, SA & Pooled & Ewes & 7 \\
\hline & Mid-North 2, SA & Pooled & Ewes & 5 \\
\hline & Adelaide Hills, SA & Individual & Ewes & 23 \\
\hline & Armidale, NSW & Single animal & Wether (uninfected control) & 4 \\
\hline & Total & & & 39 \\
\hline \multirow[t]{3}{*}{ Val2 } & Eyre Peninsula, SA & Individual & Ewes and hoggets & 32 \\
\hline & Armidale, NSW & Single animal & Wether (uninfected control) & 3 \\
\hline & Total & & & 35 \\
\hline \multirow[t]{3}{*}{ Val3 } & Lower North, SA & Pooled & Ewes and rams & 22 \\
\hline & Yorke Peninsula, SA & Individual & Ewes & 10 \\
\hline & Total & & & 32 \\
\hline \multirow[t]{2}{*}{ Val4 } & Gatton, QLD & Individual & Ewes & 22 \\
\hline & Total & & & 22 \\
\hline
\end{tabular}

particulate matter, and a subsample of the egg suspension was examined by microscopy using the 0.3-mL McMaster counting chamber, giving a lower detection level of 30 epg.

As the faecal samples collected from QLD were naturally infected with $H$. contortus, the blood content in the faeces was unknown. Furthermore, there was no available method to accurately measure the blood concentrations in the faeces. Therefore, the amount of faecal occult blood in the faeces from the QLD samples was estimated using Hemastix ${ }^{\circledR}$ (Bayer Australia, Pymble, NSW, Australia) based on a modified protocol described by Colditz and Le Jambre [18]. Briefly, two subsamples from each sample bag were diluted in water to yield a final dilution of 1:500. Samples were boiled for $20 \mathrm{~min}$ and cooled for 3-5 min in a container with tap water before testing. Hemastix ${ }^{\circledR}$ scores were obtained by dipping the reagent strips into the cooled diluted faecal mixture, and the colour change of the strip was assessed against a reference colour chart (provided on the reagent bottle) after $60 \mathrm{~s}$ to provide a score between 1 (no color change) and 5 (dark green).

The FAMACHAC scores of individual sheep were obtained by examining the colour of the lower eyelid mucous membranes and comparing it to a chart of colour standards, with a score of 1-2 representing 'not anaemic' and scores of 3-5 indicating levels of anaemia requiring treatment [22]. Of the 22 samples collected 
from Gatton, QLD, eight were collected at random with no prior knowledge of infection history or FAMACHA® score; thus, these samples were removed from the analysis. The Vis-NIR-predicted $\mathrm{Hb}$ values for the faecal samples were then compared with the results from the FWEC, Hemastix ${ }^{\circledR}$ and FAMACHA $\odot$ assessments.

\section{Chemometric analysis and statistics}

All spectral data were exported using F750 DataViewer (v 1.2) and analysed as raw spectra using The Unscrambler X (v. 10.5.1; CAMO A/S, Oslo, Norway). RStudio (v. 1.0.153; https://rstudio.com/products/rstudio/) was used to establish the plots presented here. The spectral data were pre-treated using the Savitzky-Golay filter with second derivative order smoothing, second polynomial order and seven smoothing points to remove scattering effects from the spectra prior to further analysis. Spectral measurements were initially investigated using principal component analysis (PCA) to identify spectrally similar samples, and outliers were identified using the Hotelling $T^{2}$ statistics. Spectra that had extreme spectral signals, leverage and residual means relative to other samples were considered to be outliers. The impact of sample origin (location), class of sheep, faecal consistencies and sample type on the SavitzkyGolay transformed spectra was analysed using PCA within the wavelength range of 387-609 $\mathrm{nm}$.

The calibration models for the detection of $\mathrm{Hb}$ in sheep faeces were developed using partial least squares (PLS) regression analysis with randomised blocks of cross-validation. Calibration models built in this study were confined within the wavelength range of 387$609 \mathrm{~nm}$, which contained the relevant $\mathrm{Hb}$ absorption bands [42]. The coefficient of determination for correlation in calibration $\left(R_{\text {cal }}^{2}\right)$, root-mean-square error of calibration (RMSEC), coefficient of determination for correlation in cross-validation $\left(r_{\mathrm{cv}}^{2}\right)$ and the rootmean-square error of cross-validation (RMSECV) were used to assess the model performance. The number of latent variables (LVs) was determined by the leaveone-out cross-validation method [47-49]. For analysis, FWEC was transformed using $\log _{10}($ FWEC +10$)$. A transformed epg of 2.82 (650 epg) was used as the threshold for anthelmintic treatment [43]. Prediction statistics of the validation dataset was evaluated using the coefficient of determination for correlation of prediction $\left(r_{\mathrm{p}}^{2}\right)$, and root-mean-squared error of prediction (RMSEP). Predictions are considered good if they have $r_{\mathrm{p}}^{2}>0.80$ with the smallest RMSEP. A level of $3 \mu \mathrm{g} \mathrm{Hb} / \mathrm{mg}$ faeces was used as a threshold for anthelmintic treatment to calculate the prediction accuracies for the calibration models built in this study $[42,50]$.
The sensitivity (\%SN) of the model refers to the percentage of samples correctly identified as indicating the need for treatment (> $3 \mu \mathrm{g} \mathrm{Hb} / \mathrm{mg}$ faeces), while the specificity $(\% \mathrm{SP})$ of the model refers to the percentage of the samples correctly predicted as 'healthy' samples where treatment was not needed $(<3 \mu \mathrm{g} \mathrm{Hb} / \mathrm{mg}$ faeces $)$. The formulas used for the calculation of sensitivity and specificity of the predicted results are as follows:

$$
\begin{aligned}
& \% \mathrm{SN}=\frac{\text { True positive }}{\text { True positive }+ \text { False negative }}, \\
& \% \mathrm{SP}=\frac{\text { True negative }}{\text { False positive }+ \text { True negative }} .
\end{aligned}
$$

True positive indicates samples containing $>3 \mu \mathrm{g} \mathrm{Hb} /$ mg faeces that were correctly predicted, whereas true negative indicates samples containing $<3 \mu \mathrm{g} \mathrm{Hb} / \mathrm{mg}$ faeces that were correctly predicted.

PCA was also applied to the QLD samples for analysis of the pre-processed spectra (Savitzky-Golay 1st derivative, 2nd polynomial order and 5 smoothing points) within the wavelength region of 387-609 $\mathrm{nm}$ for analysis of the loading and score plots.

\section{Results}

The raw mean-centred absorbance spectra of sheep faeces containing various $\mathrm{Hb}$ concentrations covering the range of 380-1200 nm are illustrated in Fig. 1. All faecal samples collected in this study showed a similar profile and trend. Two distinct bands were observed around 576 and $670 \mathrm{~nm}$, which had been found previously to be associated with $\mathrm{Hb}$ and chlorophyll, respectively $[42,51,52]$. Subsequently, for the prediction of $\mathrm{Hb}$ in sheep faeces in the present study, all analyses were carried out within the wavelength range of 387-609 $\mathrm{nm}$ to limit the effects of the 670-nm chlorophyll peak.

PCA of the Savitzky-Golay filtered spectra indicated that the first two principal components, PC-1 and PC-2, explained 53 and $16 \%$ of the total variance, respectively (Fig. 2). The samples appeared to primarily cluster on the basis of the location from which the faeces were collected (Fig. 2a). Samples collected from Mid-North 1 and 2 and Adelaide Hills were spectrally similar and clustered around the positive values of PC-1. Samples from the Eyre Peninsula were split into two clusters, with a majority of the samples lying in the quadrant of positive values of PC-2 along with samples from Lower North, while the remaining samples clustered in the negative values of PC-2. The separation between samples from Eyre Peninsula was associated with the difference in class of sheep; samples in the positive values of $\mathrm{PC}-2$ were associated with samples collected from ewes, while the remaining 
samples were collected from hoggets (Fig. 2b). Samples from the Fleurieu Peninsula also showed clustering; samples collected from ewes were clustered within the positive quadrant of $\mathrm{PC}-2$, while samples collected from lambs clustered within the negative quadrant of PC-2.

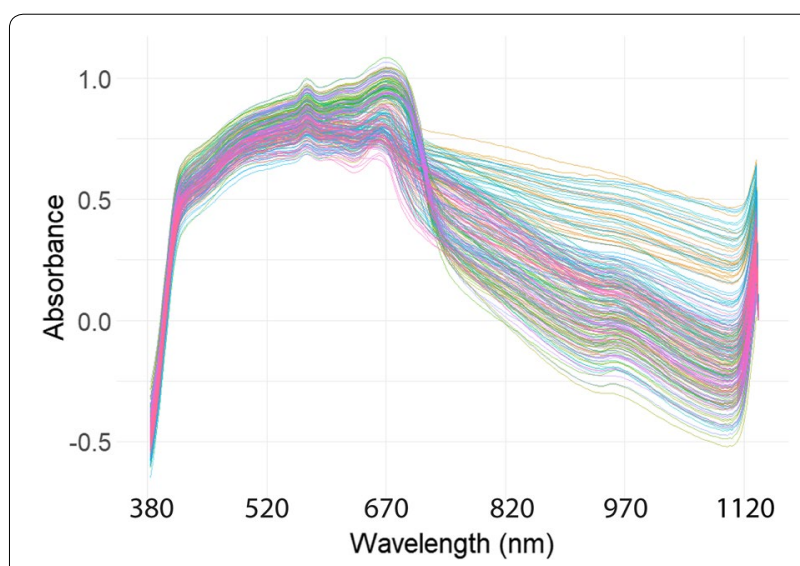

Fig. 1 Raw absorbance visible-near infrared spectra of all faecesblood mixture samples prepared for the prediction of haemoglobin in sheep faeces
Although no apparent clustering was observed when faecal samples were grouped based on the consistency of the faeces (Fig. 2c), it was found that the pooled samples clustered more closely together (Fig. 2d). In contrast, individual samples were more scattered (Fig. 2d).

Table 3 shows the prediction accuracies for the models built using PLS regression with the SavitzkyGolay-transformed spectra. The two models showed similar performance statistics of $R_{\text {cal }}^{2}=0.48-0.49$ and RMSEC $=2.26-2.27 \mu \mathrm{g} \mathrm{Hb} / \mathrm{mg}$ faeces for the prediction of $\mathrm{Hb}$ concentrations in field-collected sheep faeces. The inclusion of pooled samples in Model 2 gave a slight improvement in the performance for both calibration and cross-validation compared with Model 1, which consisted of individual samples. Still, this difference is unlikely to be significant.

The calibration models were applied to the validation datasets to predict $\mathrm{Hb}$ in faecal samples collected from various locations. Table 4 shows the prediction accuracies for validation datasets $1-3$. Prediction of $\mathrm{Hb}$ in a mixture of pooled and individual samples collected from Mid-North 1 and 2, Adelaide Hills and Armidale (Val1) showed poor prediction statistics $\left(r^{2}{ }_{\mathrm{p}}<0.05\right.$ and RMSEP $>2.78 \mu \mathrm{g} \mathrm{Hb/mg} \mathrm{faeces)} \mathrm{using} \mathrm{both} \mathrm{Model}$
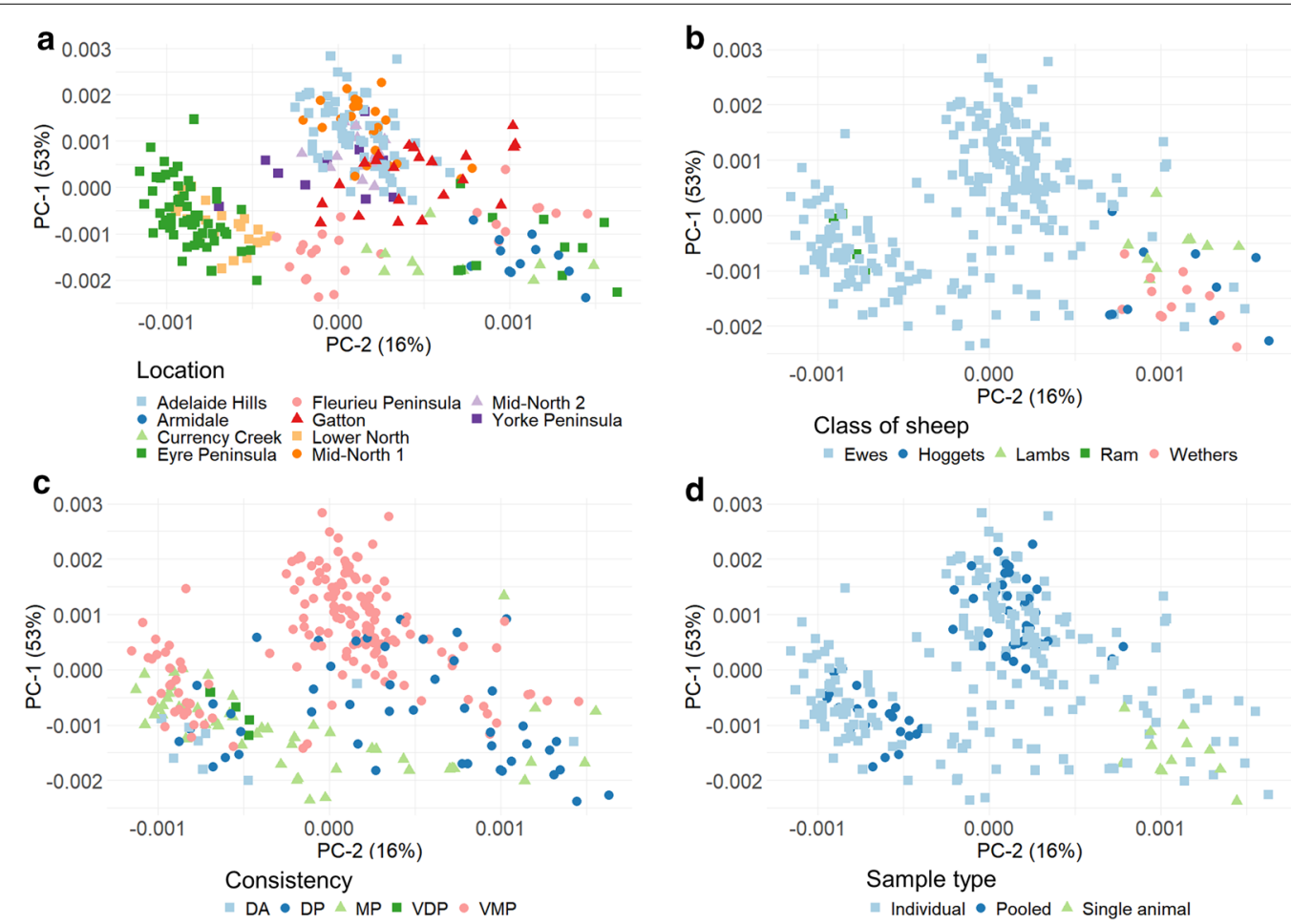

Fig. 2 Score plots of principal component analysis (PCA) for Savitzky-Golay filtered spectra of all samples measured using visible-near infrared (Vis-NIR) spectrometry within the wavelength region of 387-609 nm. Samples are grouped by location (a), class of sheep (b), faeces consistency (c) and sample type (d). DA Dry aggregates, DP dry pellets, MP moist pellets, $P C-1, P C-2$ principal components 1, 2, respectively, VDP very dry pellets, VMP very moist pellets 
1 and Model 2. In contrast, predictions of $\mathrm{Hb}$ in both pooled and individual faecal samples collected from Eyre Peninsula, Armidale, Lower North and Yorke Peninsula (Val2 and Val3) showed average prediction statistics $\left(r_{\mathrm{p}}^{2}>0.6\right.$ and RMSEP $=1.53-1.73 \mu \mathrm{g} \mathrm{Hb} / \mathrm{mg}$ faeces) using both models. Based on a threshold for treatment of $3 \mu \mathrm{g} \mathrm{Hb} / \mathrm{mg}$ faeces, high sensitivity was observed for Val1 when Model 1 and 2 were employed to predict for high $\mathrm{Hb}$ concentrations $(>3 \mu \mathrm{g} \mathrm{Hb} / \mathrm{mg}$ faeces) in the faecal samples (\% $\mathrm{SN}>71.4 \%)$. However, there was low specificity (\%SP $<66.7 \%)$ for samples with low $\mathrm{Hb}$ concentrations $(<3 \mu \mathrm{g} \mathrm{Hb} / \mathrm{mg}$ faeces $)$. The overall sensitivity and specificity of $\mathrm{Hb}$ predictions were higher for Val2 (\%SN> 82.5\%, \%SP $>72.2 \%)$ and Val3 (\%SN $>88.9 \%, \% \mathrm{SP}>64.3 \%)$ than for Val1, and $\mathrm{Hb}$ predictions performed with higher accuracies using Model 2 than Model1 (Table 4).

Figure 3 shows the relationship between $\mathrm{Hb}$ concentrations predicted using Vis-NIR spectroscopy (Models 1 and 2$)$ and $\log _{10}($ FWEC +10$)$, Hemastix ${ }^{\circledR}$ and FAMACHA $\odot$ for samples collected from QLD. The FWEC for samples collected from QLD contained between 0 epg (transformed epg $=0$ ) and 8340 epg (transformed epg $=3.93$ ), while the blood leakage levels as indicated by Hemastix ${ }^{\circledR}$ and FAMACHA@ ranged from 1 to 4 , respectively. Overall, high levels of $\mathrm{Hb}$ were predicted for all QLD samples using the Vis-NIR spectroscopy (>4 $\mu \mathrm{g} \mathrm{Hb} / \mathrm{mg}$ faeces), including samples found to contain low blood leakage based on Hemastix ${ }^{\circledR}$ and FAMA$\mathrm{CHA} \odot$ tests. On average, the Vis-NIR-predicted $\mathrm{Hb}$ values in QLD faeces were slightly lower using calibration Model 1 than Model 2. As with the samples from $\mathrm{SA}$, faecal consistency did not appear to affect the $\mathrm{Hb}$ predictions for the faeces collected from QLD.

Figure 4 shows the PCA loading plot for SavitzkyGolay pre-processed spectra of the samples collected from QLD. Bands of interest for spectra pre-processed with Savitzky-Golay first derivative typically lie in the zero plane of the loading units [53]. The first four principal components (PCs) accounted for $96 \%$ of the total spectral variation observed. Based on the PCA loading plots, the peaks at $453,465,504,534,540,555$ and
$576 \mathrm{~nm}$ contributed to the majority of the spectral variation in the naturally infected QLD samples.

Interestingly, the PCA score plots of the pre-processed spectra for all of the naturally infected QLD samples showed clustering based on their Hemastix ${ }^{\circledR}$ and FAMACHA@ scores (Fig. 5). The samples with high Hemastix ${ }^{\circledR}$ and FAMACHA® scores (> 3), indicating that anthelmintic treatment may be required, clustered in the positive quadrant of PC-1, with one potential outlier. The remaining samples with lower scores trended from the positive to the negative quadrant of PC-2.

\section{Discussion}

The development of a more effective and rapid approach for diagnosing $H$. contortus infections would have significant benefits for sheep producers. Accurate detection of the presence of $\mathrm{Hb}$ in faeces may also enable earlier diagnosis of $H$. contortus infection in sheep than is possible using existing methods as blood can be detected in the faeces approximately 7 days before $H$. contortus eggs are found [9].

In this study, the Vis-NIR models for sheep blood content successfully differentiated 'healthy' samples from those requiring anthelmintic treatment with moderate to good prediction accuracy (sensitivity $57-94 \%$, specificity $44-79 \%$ ). However, PCA indicated that the pre-processed spectra were clustered on the basis of location and the class of sheep from which the samples were collected. It has previously been demonstrated that faeces obtained from animals grazing on different rangelands and pastures vary in a number of components, including longchain alcohols, very-long-chain fatty acids and alkanes $[54,55]$, all of which will affect the absorbance of the spectra. Therefore, the separation of samples from different locations may reflect the diet consumed by the sheep, as different locations are likely to be associated with differing pasture compositions.

Differences in the faecal Vis-NIR spectra in different classes of sheep have previously been reported by Godfrey et al. [56] who found differences between male, pregnant and lactating animals [56]. The differences in the

Table 3 Prediction statistics for the calibration models built with Savitzky-Golay transformed spectra of faeces with various haemoglobin concentrations using the wavelength region of $387-609 \mathrm{~nm}$

\begin{tabular}{|c|c|c|c|c|c|c|c|}
\hline \multicolumn{2}{|c|}{ Calibration } & \multirow[t]{2}{*}{ Regression analysis } & \multirow[t]{2}{*}{ Latent variables $(N)$} & \multicolumn{2}{|c|}{ Calibration } & \multicolumn{2}{|c|}{ Cross-validation } \\
\hline Models & $N$ & & & $R_{\text {cal }}^{2}$ & $\overline{\text { RMSEC }}$ & $r_{\mathrm{cv}}^{2}$ & RMSECV \\
\hline 1 & 119 & PLS & 6 & 0.48 & 2.27 & 0.31 & 2.65 \\
\hline 2 & 153 & PLS & 6 & 0.49 & 2.26 & 0.33 & 2.59 \\
\hline
\end{tabular}

PLS, Partial least squares; $R^{2}$ cal, coefficient of determination for correlation in calibration; RMSEC, root-mean-squared error of calibration (units in $\mu \mathrm{g} H \mathrm{~b} / \mathrm{mg}$ faeces); $r^{2}$ cv, coefficient of determination for correlation in cross-validation; RMSECV, root-mean-squared error of cross-validation (units in $\mu \mathrm{g} \mathrm{Hb} / \mathrm{mg}$ faeces) 


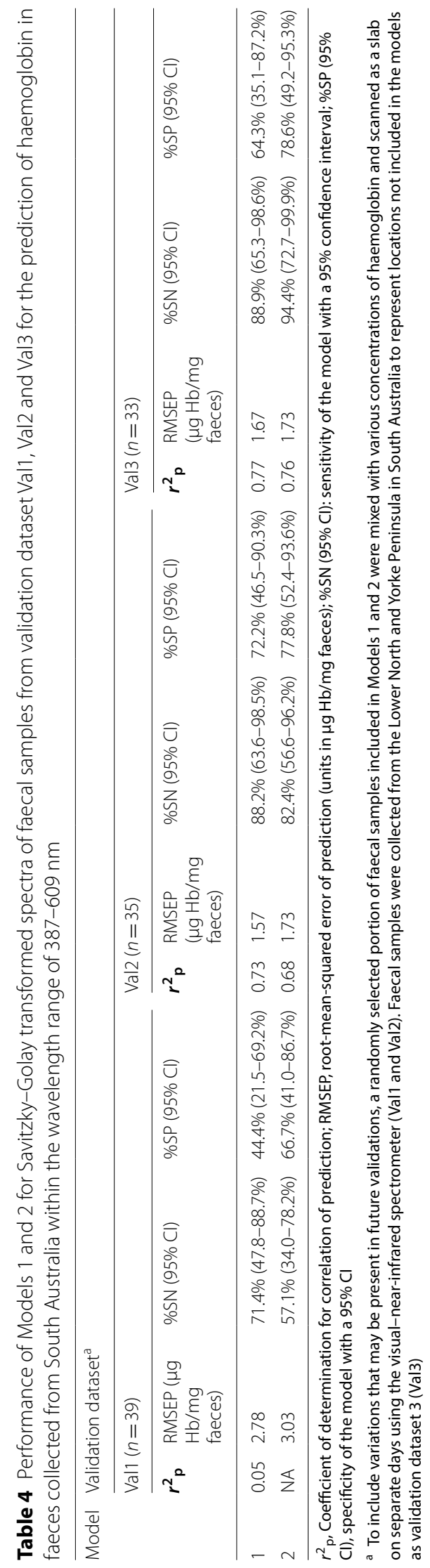




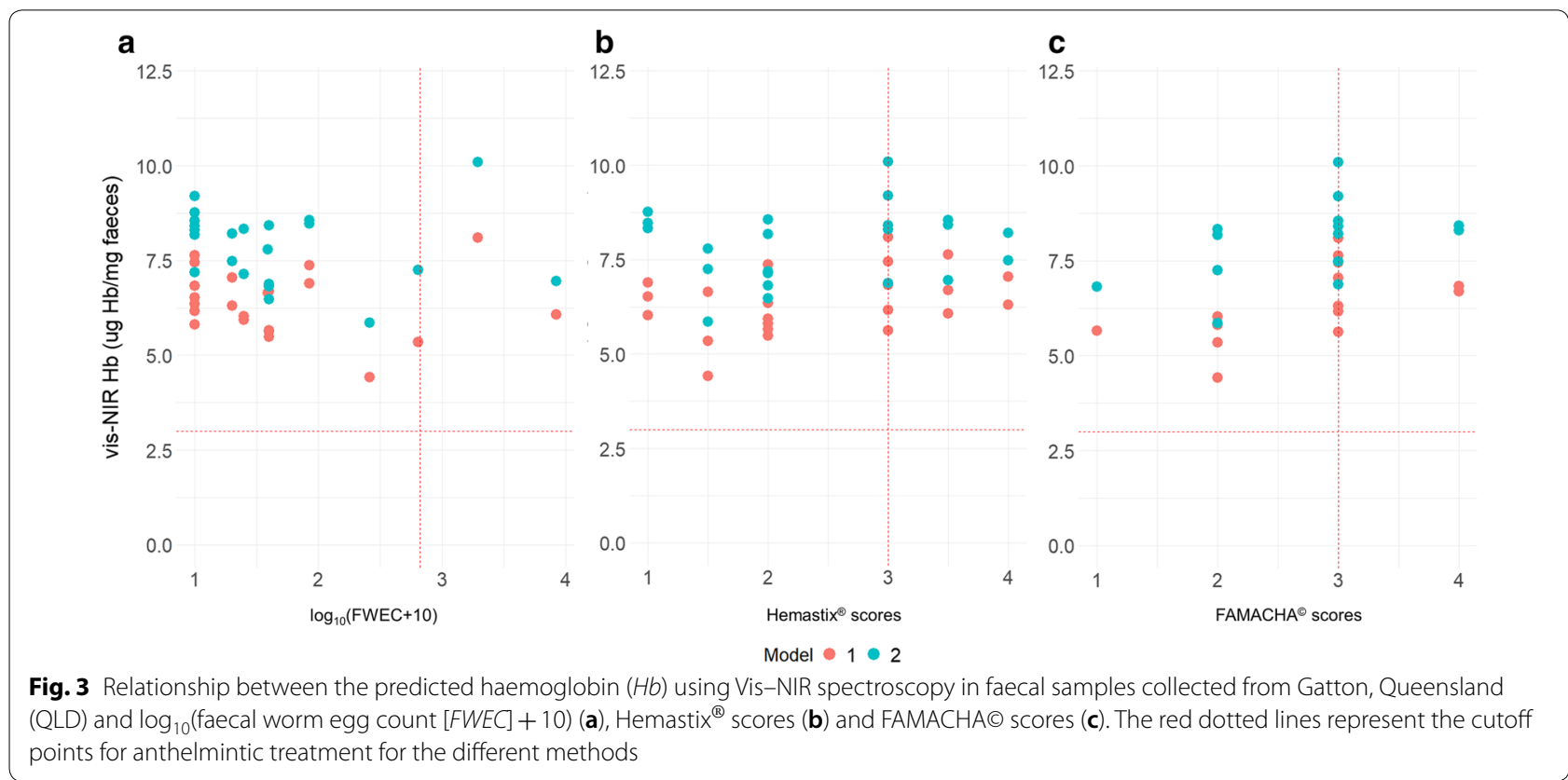

faecal Vis-NIR spectra in age classes of sheep seen in our study are not surprising and may be related to differences in maturity of the digestive systems between younger and older sheep, which have been previously shown to affect the faecal NIR spectra from goats [57]. No clear separation was seen due to the faecal consistency (Fig. 2c), which suggests that the moisture of the faecal samples did not affect the prediction accuracy of models established using the wavelength region of $387-609 \mathrm{~nm}$. This is consistent with the results from our previous study

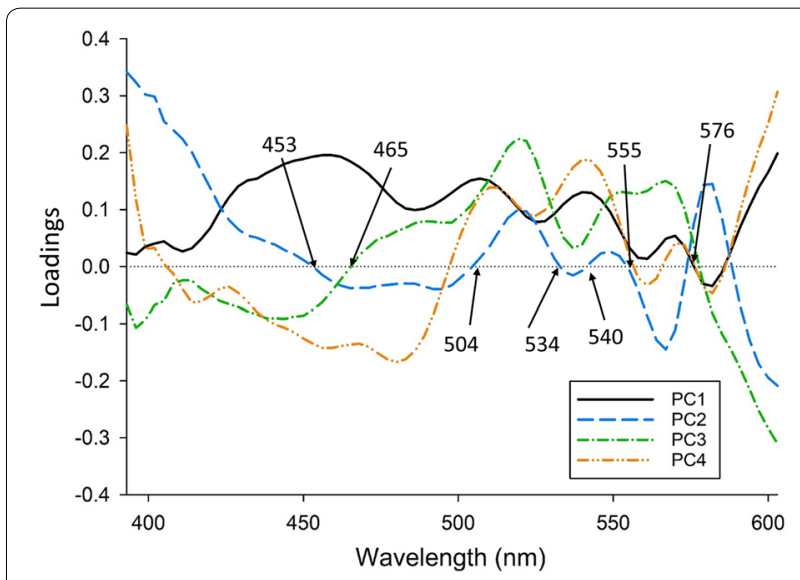

Fig. 4 PCA loading plot of the Savitzky-Golay (1st derivative order, 2nd polynomial order and 5 smoothing points) within the wavelength range of 387-609 $\mathrm{nm}$ for sheep faeces naturally infected with Haemonchus contortus collected from Queensland. PC1-PC4 Principal components $1-4$ which showed that $\mathrm{Hb}$ concentrations in sheep faeces were accurately predicted regardless of whether the samples were dried or not prior to scanning [42]. Although samples prepared for NIR spectroscopy often require drying to avoid interference from moisture [38, 39], our results suggest that moisture content does not affect the prediction of $\mathrm{Hb}$ concentrations in sheep, eliminating the need to dry faecal samples for on-farm application.

Pooled faecal samples clustered more closely together than individual samples (Fig. 2d). These samples were thoroughly homogenised before adding blood to the faeces, resulting in an evenly mixed faeces-blood mixture with minimal variation between samples. The use of pooled faecal samples for faecal NIR analysis has also been recommended for the prediction of diet qualities in livestock [58] as the nutritional diet backgrounds of animals grazing in the same area are similar enough to provide a level of precision adequate for herd management [59]. Our results show that the inclusion of pooled samples in the calibration model did not significantly affect the prediction statistics of the prediction models. This result has important implications, because pooled sample collection is far more practical than the use of individual samples for parasite surveillance in large sheep flocks, and is also the recommended method for sampling FWEC to determine the need for flock anthelmintic treatments [12]. The inclusion of a mixture of pooled and individual samples in a calibration model will assist in capturing the majority of the variations observed between individuals in a mob of sheep, thus improving the robustness of the prediction models developed. 


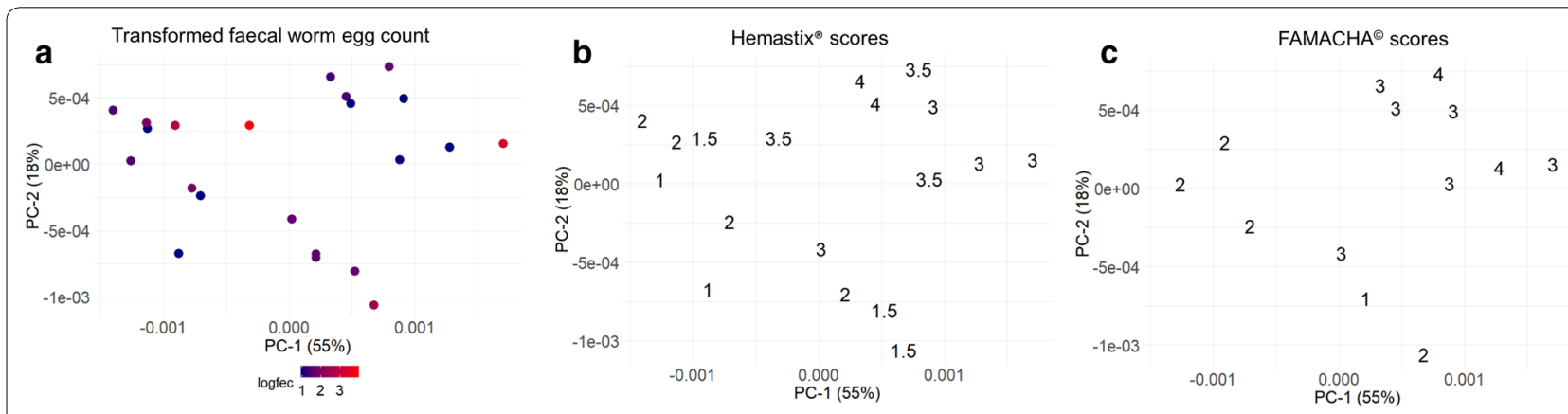

Fig. 5 Score plots of PC-1 and PC-2 from the PCA performed within the region of 387-609 nm for the visible-near-infrared spectra of faecal samples collected from Gatton, QLD. Samples were pre-processed using Savitzky-Golay derivative smoothing and grouped based on log ${ }_{10}$ (faecal worm egg count +10$)(\mathbf{a})$, Hemastix ${ }^{\circledR}$ scores $(\mathbf{b})$ and FAMACHA@ scores $(\mathbf{c})$

The $\mathrm{Hb}$ predictions for samples in Val1 showed lower accuracy than the other datasets. Samples in Val1 were freeze-thawed multiple times, and while it is not known how the freeze-thaw process affects the chemical composition of faeces, we have previously observed difference in spectra between samples stored at $4{ }^{\circ} \mathrm{C}$ and those stored at $-20{ }^{\circ} \mathrm{C}$ (unpublished data), suggesting that the temperature may have been a factor. In contrast, the $\mathrm{Hb}$ for samples in Val2 were predicted with a high level of accuracy using models established with both individual (Model 1) and mixed pooled and individual samples (Model 2). Although a higher $r^{2}$ and lower RMSEP prediction statistics with Model 1 indicated slightly better $\mathrm{Hb}$ predictions for samples in Val2 compared to Model 2 , the sensitivity and specificity of the predicted $\mathrm{Hb}$ were higher for these samples with Model 2 (mixed pooled and individual samples) than Model 1. Similarly, sensitivity and specificity based on a threshold for treatment at $3 \mu \mathrm{g} \mathrm{Hb} / \mathrm{mg}$ faeces were higher for the prediction of $\mathrm{Hb}$ in samples from Val3 using Model 1 than with Model 2. This suggests that the inclusion of pooled samples in the calibration model may improve prediction of the need for anthelmintic treatment, which is in line with the recommendations provided for using faecal NIR spectroscopy to estimate nutritional profiles in livestock [58].

Although the locations of origin of faecal samples in Val3 (Lower North and Yorke Peninsula) were not represented in either of the calibration models, high accuracies for the prediction of $\mathrm{Hb}$ were observed for these samples using both Model 1 and 2. This may be due in part to similarities in faecal composition of samples, as these samples originate from similar climatic zones of SA with similar sheep management and likely similar pasture compositions between the two areas.

In contrast, validation using samples collected from QLD did not show any relationship with FWEC, Hemastix ${ }^{\circledR}$ scores and FAMACHA® scores (Fig. 3) and all samples collected from QLD were predicted to have high $\mathrm{Hb}$ concentrations. This may be due to a number of factors. The low precision may have been related to the difference in faecal composition between the samples collected in SA and QLD, which may be due in part to differences in climate or the pastures on which the sheep were grazing. Additionally, the calibration models were built based on spiked samples from SA for which the concentration of blood was accurately known. The amount of blood in the faeces from QLD samples was unknown and estimated using Hemastix ${ }^{\circledR}$ and FAMACHA® scores. Thirdly, of the peaks observed from the PCA loading plots for QLD samples, only two peaks had been previously found to be associated with blood. The two peaks, at 540 and $576 \mathrm{~nm}$, respectively, which are associated with oxyhemoglobin [60, 61], were observed in our study with spiked blood [42]. In contrast, the peak at $555 \mathrm{~nm}$ was not previously observed and has been shown to be due to deoxyhemoglobin [60]. Therefore, the lower precision for the prediction of $\mathrm{Hb}$ in QLD samples could also be due in part to the difference in blood chemicals between naturally occurring FOB and the blood used to spike the faeces in the calibration models.

Interestingly, when PCA was performed on the preprocessed spectra, we found that the QLD faecal samples showed separation between samples with high Hemastix $^{\circledR}$ and FAMACHA@ scores $(>3)$ and those with lower scores. This result confirms that the collected Vis-NIR spectra within the wavelength range of $387-609 \mathrm{~nm}$ for QLD samples were correlated at some level to blood loss found in the faecal samples.

The results from PCA did not show any clear relationship between Vis-NIR-predicted $\mathrm{Hb}$ concentrations and the transformed FWEC, which may be due to the fact that $H$. contortus eggs are typically detected around 1 week after the presence of blood in sheep faeces $[18,62]$ and may not have been present in some or all of the sheep at the time of sampling. Results from this 
experiment suggest that further research to expand the calibration model by including faecal samples collected from a range of areas in QLD that are naturally infected with $H$. contortus is necessary.

\section{Conclusion}

The calibration models built in this study using faeces spiked with sheep blood were useful in predicting $\mathrm{Hb}$ for faecal samples within the same geographical region and provided further evidence for the feasibility of using Vis-NIR spectroscopy for the diagnosis of $H$. contortus infections in sheep. The location where the faecal samples were collected, the class of sheep and the type of faecal samples (pooled or individual) were significant factors affecting the predictability of the calibration model. The prediction sensitivity and specificity based on the anthelmintic treatment threshold ( $3 \mu \mathrm{g} \mathrm{Hb} / \mathrm{mg}$ faeces) for both pooled and individual samples were high, particularly when using a calibration model built on a mixture of pooled and individual animals. Although the models built using blood-spiked faecal samples from sheep in SA were not predictive for samples collected from QLD, we demonstrated that Vis-NIR spectroscopy shows high potential for determining which sheep or mob of sheep require treatment. Furthermore, this is the first study to identify the peaks within the visible range for FOB found in naturally infected sheep faeces, which will provide substantial insight for the future development of Vis-NIR models for predicting FOB. To further develop a field-ready Vis-NIR calibration model, future work should focus on using faeces collected from naturally infected animals across a wide range of environments and sheep types in Haemonchus-endemic areas.

\begin{abstract}
Abbreviations
DA: Dry aggregate; DP: Dry pellet; epg: Eggs per gram; FOB: Faecal occult blood; FWEC: Faecal worm egg count; GIN: Gastrointestinal nematode; $\mathrm{Hb}$ : Haemoglobin; LVs: Latent variables; MP: Moist pellet; NIR: Near-infrared; NSW: New South Wales; PCA: Principal component analysis; PC: Principal component; PLS: Partial least squares; $R^{2}$ cal: Coefficient of determination for correlation in calibration; QLD: Queensland; RMSEC: Root-mean-square error of calibration; $r_{\mathrm{cv}}^{2}$ : Coefficient of determination for correlation in cross-validation; RMSECV: Root-mean-square error of cross-validation; $r^{2}$ : Coefficient of determination for correlation of prediction; RMSEP: Root-mean-squared error of prediction; SA: South Australia; VDP: Very dry pellet; VMP: Very moist pellet; Vis-NIR: Visible-near-infrared; \%SN: Sensitivity in percentage; \%SP: Specificity in percentage.
\end{abstract}

\section{Acknowledgements}

The authors gratefully acknowledge Peter Hunt and Marielle Babineau at the CSIRO FD McMaster laboratory, Armidale, NSW, for kindly supplying the faecal samples from NSW used in the present study.

\section{Authors' contributions}

EAK, JNF, ACK, GPF, MS, AMB, SSM and PJJ discussed the contents of the research. EAK, PJJ and AMB collected the samples. EAK carried out all the experiments and spectra collection and performed the spectral data analysis.
EAK drafted the initial manuscript. All authors contributed their expertise and advice to produce the final version of the manuscript. All authors have read and approved the final manuscript.

\section{Funding}

EAK was supported by The University of Queensland Peel Fellowship during the conduct of this work.

\section{Availability of data and materials \\ Not applicable.}

\section{Ethics approval and consent to participate}

All animal procedures were approved by the FD McMaster Animal Ethics Committee, CSIRO Agriculture and Food (Animal Ethics Approval Number: AEC 18/09) and The University of Queensland Animal Ethics Committee (Animal Ethics Approval Number: SVS/452/17).

\section{Consent for publication}

Not applicable.

\section{Competing interests}

The authors declared no potential conflicts of interest with respect to the research, authorship, and/publication of this article.

\section{Author details}

${ }^{1}$ The Queensland Alliance for Agriculture and Food Innovation, The University of Queensland, St. Lucia, QLD 4067, Australia. ${ }^{2}$ Commonwealth Scientific and Industrial Research Organisation (CSIRO) Agriculture and Food, St. Lucia, QLD 4067, Australia. ${ }^{3}$ Department of Food Science and Technology, University of California, Davis, CA 95616, USA. ${ }^{4}$ The School of Public Health, The University of Queensland, Herston, Queensland 4006, Australia. ${ }^{5}$ The School of Agriculture \& Food Sciences, The University of Queensland, Gatton, QLD 4343, Australia.

Received: 20 July 2020 Accepted: 5 November 2020

Published online: 23 November 2020

\section{References}

1. Lane J, Jubb T, Shepherd R, Webb-Ware J, Fordyce G. Priority list of endemic diseases for the red meat industries. Final Report. Meat \& Livestock Australia. 2015. https://www.mla.com.au/research-and-devel opment/search-rd-reports/final-report-details/Animal-Health-and-Biose curity/Priority-list-of-endemic-diseases-for-the-red-meat-industries/2895. Accessed 13 May 2020.

2. Anderson RC. Nematode parasites of vertebrates: their development and transmission. 2nd ed. Wallingford: CABI Publishing; 2000.

3. Brightling A, Brightling T, Fowler D. Livestock diseases in Australia: diseases of cattle, sheep, goats and farm dogs. Mt Waverley: C.H. Jerram \& Associates; 2006

4. Besier R, Dunsmore J. The ecology of Haemonchus contortus in a winter rainfall region in Australia: the development of eggs to infective larvae. Vet Parasitol. 1993;45(3-4):275-92.

5. O'Connor LJ, Walkden-Brown SW, Kahn LP. Ecology of the free-living stages of major trichostrongylid parasites of sheep. Vet Parasitol. 2006;142(1):1-15.

6. Emery DL, Hunt PW, Le Jambre LF. Haemonchus contortus: the then and now, and where to from here? Int J Parasitol. 2016;46(12):755-69.

7. Gordon HM. The epidemiology of parasitic diseases, with special reference to studies with nematode parasites of sheep. Aust Vet $J$ 1948:24(2):17-45.

8. Southcott W, Major G, Barger I. Seasonal pasture contamination and availability of nematodes for grazing sheep. Crop Pasture Sci. 1976;27(2):277-86.

9. Albers G, Le Jambre L. Erythrocyte potassium concentration: a simple parameter for erythropoiesis in sheep infected with Haemonchus contortus. Res Vet Sci. 1983;35(3):273-6. https://doi.org/10.1016/S0034 $-5288(18) 32018-6$.

10. Bowman DD. Georgis' parasitology for veterinarians: helminths. Edinburgh: Elsevier Health Sciences; 2014. 
11. Colditz IG. Challenges to the development of new tests for diagnosis of infection and prediction of resistance of sheep to gastrointestinal nematodes. Trop Biomed. 2008;25(1):41-9.

12. Coles G, Bauer C, Borgsteede F, Geerts S, Klei T, Taylor M, et al. World Association for the Advancement of Veterinary Parasitology (WAAVP) methods for the detection of anthelmintic resistance in nematodes of veterinary importance. Vet Parasitol. 1992;44(1-2):35-44.

13. Preston SJM, Sandeman M, Gonzalez J, Piedrafita D. Current status for gastrointestinal nematode diagnosis in small ruminants: where are we and where are we going? J immunol Res. 2014. https://doi. org/10.1155/2014/210350.

14. El-Abdellati A, Charlier J, Geldhof P, Levecke B, Demeler J, von SamsonHimmelstjerna $G$, et al. The use of a simplified faecal egg count reduction test for assessing anthelmintic efficacy on Belgian and German cattle farms. Vet Parasitol. 2010;169(3-4):352-7.

15. Hunt P, Lello J. How to make DNA count: DNA-based diagnostic tools in veterinary parasitology. Vet Parasitol. 2012;186(1):101-8.

16. Levecke B, Rinaldi L, Charlier J, Maurelli M, Bosco A, Vercruysse J, et al. The bias, accuracy and precision of faecal egg count reduction test results in cattle using McMaster, Cornell-Wisconsin and FLOTAC egg counting methods. Vet Parasitol. 2012;188(1-2):194-9.

17. Woolaston R, Baker R. Prospects of breeding small ruminants for resistance to internal parasites. Int J Parasitol. 1996;26(8-9):845-55.

18. Colditz IG, Le Jambre LF. Development of a faecal occult blood test to determine the severity of Haemonchus contortus infections in sheep. Vet Parasitol. 2008;153(1):93-9.

19. Simon JB. Occult blood screening for colorectal carcinoma: a critical review. Gastroenterology. 1985;88(3):820-37.

20. Gaspar T, Penel C, Thorpe T, Greppin H. Peroxidases 1970-1980. A survey of their biochemical and physiological roles in higher plants. Geneva: University of Geneva; 1982.

21. Rodríguez A, Goldberg V, Viotti H, Ciappesoni G. Early detection of Haemonchus contortus infection in sheep using three different faecal occult blood tests. Open Vet J. 2015;5(2):90-7.

22. Bath G, Malan F, Van Wyk J. The "FAMACHAC" ovine anaemia guide to assist with the control of haemonchosis. In: Proc 7th Annual Congress of the Livestock Health and Production Group of the South African Veterinary Association. Port Elizabeth, South Africa, 5-7 June 1996. p. 5.

23. Burke J, Kaplan R, Miller J, Terrill T, GetzW, Mobini S, et al. Accuracy of the FAMACHAC system for on-farm use by sheep and goat producers in the southeastern United States. Vet Parasitol. 2007;147(1):89-95.

24. Di Loria A, Veneziano V, Piantedosi D, Rinaldi L, Cortese L, Mezzino L, et al. Evaluation of the FAMACHA@ system for detecting the severity of anaemia in sheep from southern Italy. Vet Parasitol. 2009;161(1-2):53-9.

25. Kaplan R, Burke J, Terrill T, Miller J, Getz W, Mobini S, et al. Validation of the FAMACHAC eye color chart for detecting clinical anemia in sheep and goats on farms in the southern United States. Vet Parasitol. 2004;123(1):105-20.

26. Reynecke DP, Van Wyk JA, Gummow B, Dorny P, Boomker J. Validation of the FAMACHA@ eye colour chart using sensitivity/specificity analysis on two South African sheep farms. Vet Parasitol. 2011;177(3-4):203-11.

27. Van Wyk J. Production trials involving use of the FAMACHAC system for haemonchosis in sheep: preliminary results. Onderstepoort J Vet Res. 2008;75(4):331-45.

28. Kim T, Choi SH, Lambert-Cheatham N, Xu Z, Kritchevsky JE, Bertin F-R, et al. Toward laboratory blood test-comparable photometric assessments for anemia in veterinary hematology. J Biomed Opt. 2016;21(10):107001.

29. Mahieu M, Aumont G. Effects of sheep and cattle alternate grazing on sheep parasitism and production. Trop Anim Health Prod. 2009;41(2):22939. https://doi.org/10.1007/s11250-008-9180-z.

30. Molento M, Gavião A, Depner R, Pires C. Frequency of treatment and production performance using the FAMACHA@ method compared with preventive control in ewes. Vet Parasitol. 2009;162(3-4):314-9.

31. Decruyenaere V, Froidmont E, Bartiaux-Thill N, Buldgen A, Stilmant D. Faecal near-infrared reflectance spectroscopy (NIRS) compared with other techniques for estimating the in vivo digestibility and dry matter intake of lactating grazing dairy cows. Anim Feed Sci Technol. 2012;173(3):220-34.

32. Huntington $\mathrm{G}$, Leonard $\mathrm{E}$, Burns J. Use of near-infrared reflectance spectroscopy to predict intake and digestibility in bulls and steers. J Anim Sci. 2011;89(4):1163-6.
33. Landau S, Dvash L, Roudman M, Muklada H, Barkai D, Yehuda Y, et al. Faecal near-IR spectroscopy to determine the nutritional value of diets consumed by beef cattle in east Mediterranean rangelands. Animal. 2016;10(2):192-202.

34. Mahipala MK, Krebs G, McCafferty P, Naumovski T, Dods K, Stephens R. Predicting the quality of browse-containing diets fed to sheep using faecal near-infrared reflectance spectroscopy. Anim Prod Sci. 2010;50(10):925-30.

35. Dixon R, Colditz I, LeJambre L, Lyndal-Murphy M, Besier B. Potential of near infrared reflectance (NIR) spectroscopy of faeces to estimate the internal parasite burden of sheep. In: Proc NIR 2013 - 16th International Conference on Near Infrared Spectroscopy, 2-7 June 2013, La GrandeMotte, France. p. 535.

36. Tolleson D, Teel P, Stuth J, Strey O, Welsh T, Carstens G. Fecal NIRS: detection of tick infestations in cattle and horses. Vet Parasitol. 2007;144(1):146-52.

37. Abbas O, Dardenne P, Baeten V. Near-infrared, mid-infrared, and Raman spectroscopy. In: Pico Y, editor. Chemical analysis of food: techniques and applications, vol. 3. 2nd ed. London: Academic Press; 2012. p. 77-134.

38. Siesler HW. Basic principles of near-infrared spectroscopy. Handbook of near-infrared analysis. Baton Rouge: CRC Press; 2007. p. 25-38.

39. Siesler HW, Ozaki Y, Kawata S, Heise HM. Near-infrared spectroscopy: principles, instruments, applications. New York: Wiley; 2008.

40. McCoy M, Edgar H, Kenny J, Gordon A, Dawson L, Carson A. Evaluation of on-farm faecal worm egg counting in sheep. Vet Rec. 2005;156(1):21-3.

41. Morgan E, Cavill L, Curry G, Wood R, Mitchell E. Effects of aggregation and sample size on composite faecal egg counts in sheep. Vet Parasitol. 2005;131(1):79-87.

42. Kho EA, Fernandes JN, Kotze AC, Fox GP, Lord M, Beasley AM, et al. Visiblenear infrared spectroscopy for detection of blood in sheep faeces. J Near Infrared Spec. 2020. https://doi.org/10.1177/0967033520927519.

43. Kho EA, Fernandes JN, Kotze AC, Sikulu-Lord MT, Fox GP, Beasley AM, et al. Detection of Haemonchus contortus nematode eggs in sheep faeces using near and mid-infrared spectroscopy. J Near Infrared Spec. 2020. https://doi.org/10.1177/0967033520924491.

44. Besier B. Re-thinking the summer drenching program. J Agric West Aust. 2001;42(1):6-9.

45. Australian Government Bureau of Meteorology. University of Queensland Gatton (040082) Mar 2020 rainfall. https://www.bom.gov.au/jsp/ncc/ cdio/weatherData/av?p_display_type $=$ dataDGraph\&p_stn_num $=04008$ $2 \&$ p_nccObsCode $=136 \&$ p_month $=02 \&$ p_startYear $=2020$. Accessed 11 May 2020.

46. Love S. WormTest for livestock and guide to egg counts. In: Primefact 480 https://www.dpi.nsw.gov.au/_data/assets/pdf_file/0016/110077/wormt est-for-livestock-and-guide-to-egg-counts.pdf. New South Wales Department of Primary Industries, 2007. Accessed 2 Apr 2020.

47. Abdi H. Partial least square regression (PLS regression). Encycl Res Methods Soc Sci. 2003;6(4):792-5.

48. Martens H, Naes T. Multivariate calibration. Chichester: Wiley; 1992.

49. Wold S, Martens $H$, Wold $H$. The multivariate calibration problem in chemistry solved by the PLS method. In: In: Kågström B, Ruhe A, editors. Matrix pencils. New York: Springer; 1983. p. 286-93.

50. The University of New England NSW, Department of Primary Industries CSIRO, Australian Sheep Industry CRC. Development of a dipstick method for on-farm diagnosis of Haemonchus infections in ruminants. 2007. https ://www.mla.com.au/research-and-development/reports/2014/devel opment-of-a-dipstick-method-for-on-farm-diagnosis-of-haemonchus infections-in-ruminants/. Accessed 26 May 2020.

51. Chao K, Nou X, Liu Y, Kim M, Chan D, Yang C-C, et al. Detection of fecal/ ingesta contaminants on poultry processing equipment surfaces by visible and near-infrared reflectance spectroscopy. Appl Eng Agric. 2008;24(1):49-55.

52. Israsena NA, Ayudhya T, Posey FT, Tyus JC, Dingra NN. Using a microscale approach to rapidly separate and characterize three photosynthetic pigment species from fern. J Chem Educ. 2015;92(5):920-3.

53. Rinnan $\AA$, Berg FVD, Engelsen SB. Review of the most common preprocessing techniques for near-infrared spectra. TrAC Trends Anal Chem. 2009;28(10):1201-22. https://doi.org/10.1016/j.trac.2009.07.007.

54. Coates DJ, Dixon KW. Current perspectives in plant conservation biology. Collingwood: CSIRO Publishing; 2007. 
55. Dove H, Mayes R. Using n-alkanes and other plant wax components to estimate intake, digestibility and diet composition of grazing/browsing sheep and goats. Small Rumin Res. 2005;59(2-3):123-39.

56. Godfrey R, Dodson R, Bultman J, Tolleson D, Stuth J, Norman A. Use of near infrared reflectance spectroscopy to differentiate pregnancy status and gender of hair sheep in the tropics. J Anim Sci. 2001;79(Suppl 1):26.

57. Walker J, Campbell E, Lupton C, Taylor C, Waldron D, Landau S. Effects of breed, sex, and age on the variation and ability of fecal near-infrared reflectance spectra to predict the composition of goat diets. J Anim Sci. 2007;85(2):518-26.

58. White I, Hunt L, Poppi D, Petty S. Sampling requirements for predicting cattle diet quality using faecal near-infrared reflectance spectroscopy (F. NIRS) in heterogeneous tropical rangeland pastures. Rangel J. 2010:32(4):435-41.

59. Tolleson D, Schafer D. Application of fecal near-infrared spectroscopy and nutritional balance software to monitor diet quality and body condition in beef cows grazing Arizona rangeland. J Anim Sci. 2014;92(1):349-58.
60. Robles FE, Chowdhury S, Wax A. Assessing hemoglobin concentration using spectroscopic optical coherence tomography for feasibility of tissue diagnostics. Biomed Opt Express. 2010;1(1):310-7.

61. Windham WR, Lawrence KC, Park B, Buhr RJ. Visible/NIR spectroscopy for characterizing fecal contamination of chicken carcasses. Trans ASAE. 2003;46(3):747.

62. Le Jambre L. Relationship of blood loss to worm numbers, biomass and egg production in Haemonchus infected sheep. Int J Parasitol. 1995;25(3):269-73.

\section{Publisher's Note}

Springer Nature remains neutral with regard to jurisdictional claims in published maps and institutional affiliations.
Ready to submit your research? Choose BMC and benefit from:

- fast, convenient online submission

- thorough peer review by experienced researchers in your field

- rapid publication on acceptance

- support for research data, including large and complex data types

- gold Open Access which fosters wider collaboration and increased citations

- maximum visibility for your research: over $100 \mathrm{M}$ website views per year

At BMC, research is always in progress.

Learn more biomedcentral.com/submissions 